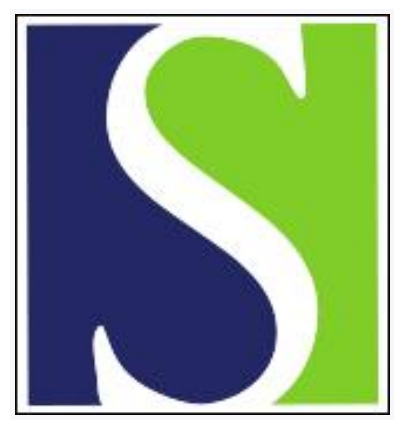

Scand J Work Environ Health 2006;32(4):294-299

https://doi.org/10.5271/sjweh.1013

Issue date: 31 Aug 2006

\title{
Recall of prior musculoskeletal pain
}

by Miranda H, Gold JE, Gore R, Punnett L

Affiliation: Department of Work Environment, University of Massachusetts Lowell, One University Avenue, Lowell, MA, USA. Helena_Miranda@uml.edu

Refers to the following text of the Journal: 1995;21(6):450-459

The following articles refer to this text: 2008;34(6):411-419;

2011;37(1):37-44; 2011;37(6):502-511; 2012;38(5):436-446;

2012;38(6):568-576; 2018;44(1):96-105; 2018;44(1):47-57

Key terms: epidemiology; memory; musculoskeletal pain; musculoskeletal symptom; recall; recall bias; self-assessment; self-report; working population

This article in PubMed: www.ncbi.nlm.nih.gov/pubmed/16932827

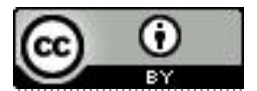




\title{
Recall of prior musculoskeletal pain
}

\author{
by Helena Miranda, MD, ${ }^{1}$ Judith E Gold, ScD, ${ }^{1,2}$ Rebecca Gore, ScD, ${ }^{1}$ Laura Punnett, $S c D^{1}$
}

\author{
Miranda H, Gold JE, Gore R, Punnett L. Recall of prior musculoskeletal pain. Scand $J$ Work Environ Health \\ 2006;32(4):294-300.
}

Objectives Patients, as well as healthy people, often fail to remember prior symptoms, illnesses, or treatments. The authors investigated how well people in a workplace recalled their prior musculoskeletal pain and which factors influenced recall accuracy.

Methods In this prospective study (1992-1998), among a cohort of automobile manufacturing workers $(\mathrm{N}=464)$, musculoskeletal pain reported at baseline was compared with recalled pain at follow-up. Two outcomes (ie, forgetting and over-recalling) were examined in relation to several personal and occupational characteristics.

Results Of those who had experienced pain or discomfort in the upper extremities at baseline, $72 \%$ did not recall it 6 years later. Symptoms at the time of recall strongly influenced pain recall; forgetting approached $100 \%$ among those with no current or recent symptoms. However, forgetting was considerable even among those with current symptoms (45\%). Prior pain status was over-recalled by $37 \%$ of those with upper-extremity pain at the time of recall, but only by $3 \%$ of those without symptoms. Women, those with history of an upperextremity disorder or systemic disease, who were clinical cases or had more anatomical areas in pain at baseline forgot less often. Over-recalling was related to age, having current symptoms, or being a clinical case. The results were similar for low-back and knee pain.

Conclusions Prior musculoskeletal symptoms are poorly remembered after some years, and the recall is strongly influenced by current symptoms. Recalled information should not be relied upon when an epidemiologic case definition is being constructed or when an attempt is being made to describe the natural history of a disorder.

Key terms epidemiology; memory; musculoskeletal symptoms; recall bias; self-reports; working population.

Pain is a person's subjective experience encompassing physiological, emotional, behavioral, and cognitive components, and hence it is difficult to measure pain objectively. In epidemiologic research, self-administered questionnaires and interviews provide a key source of information in assessments of the occurrence of pain at a population level. In addition, in everyday clinical settings, physicians are dependent on patients' self-reports when making diagnoses and determining future treatment or when evaluating the effectiveness of treatment in pain-related conditions. Despite the extensive use of retrospective self-reports, it is, however, evident that patients, as well as healthy people, unfortunately often fail to remember their prior symptoms, illnesses, or treatments.

The accuracy of the recall of pain has been a subject of several studies, the majority of which have been clinical or experimental with a relatively short recall period focusing on pain intensity among chronic pain patients (1-8). There is some inconsistency in the results regarding how well patients are able to recall their prior pain. Most of such studies have found retrospective assessment to have poor validity $(1-5,7)$, but some of the literature indicates reasonable or even good accuracy of recall $(6,8)$. The accuracy of recall is dependent on several matters, as two recent reviews concluded, such as the characteristics of the event being recalled (eg, acute or chronic pain), the length of the time period since the event, and the methods used to assess symptoms $(9,10)$. The location of pain seems to be better remembered than the intensity or frequency of pain (11). Some individual characteristics, such as age, education level, current emotional state, perception of one's own health, pain-related beliefs, and some personality traits such as negative affectivity may affect the reliability of recall. A consistent finding in earlier studies has been

1 Department of Work Environment, University of Massachusetts Lowell, Lowell, Massachusetts, United States.

2 Department of Public Health, Temple University, Philadelphia, Pennsylvania, United States.

Requests for reprints: Dr H Miranda, Department of Work Environment, University of Massachusetts Lowell, One University Avenue, Lowell, MA, USA. [E-mail: Helena_Miranda@uml.edu] 
the influence of present pain on recall; the more intense pain is at the time of recall, the more often prior pain experience is overestimated, and, conversely, if pain is presently nonintense or nonexistent, prior symptoms are often underestimated $(1,12,13)$.

Chronic pain patients differ, however, from the general population, and even more from workplace populations, which tend to be healthier than the general population. If patients with significant complaints relatively easily fail to provide accurate information on their condition, it is also important to recognize the magnitude of such a phenomenon among those who are less affected by their symptoms. Our literature search revealed only one study assessing the validity of retrospective self-reports of pain intensity in a workplace setting (13). This study found retrospective assessment of musculoskeletal pain intensity to be reliable, at least for a period of 3 months.

Our aims were to investigate how well people in a workplace recall their prior musculoskeletal pain and to determine which factors, either at baseline or at followup, influence the accuracy of recall.

\section{Study population and methods}

A prospective study was carried out between 1992 to 1998 among a fixed cohort of automobile manufacturing workers. A total of 1214 workers [46.5 (SD 8.2) years of age, $81.6 \%$ male] participated in the study, which consisted of a standardized interviewer-administered questionnaire, as well as a structured physical examination $(14,15)$. Baseline data were collected from most of the participants in 1992 (workers from one engine plant were interviewed and examined in 1993). Six years later in 1998, 914 persons still actively employed were eligible for follow-up, and 800 of them were at work during the data collection period. By the end of 1998, 538 workers were re-interviewed and re-examined, representing $67 \%$ of those actively working at the time of the follow-up.

Individual factors (eg, height, weight, prior injuries, relevant systemic diseases, alcohol and tobacco use, physical activity), as well as work-related physical and psychosocial exposures, were assessed by interview at baseline and in the follow-up. Also at baseline and in the follow-up, the participants were asked if they had experienced pain or discomfort (pain, aching, burning, stiffness, cramping, soreness, numbness, or tingling) in the upper extremity (neck, shoulder or upper arm, elbow or forearm, wrist or hand), low back, or knee that had occurred more than three times or lasted more than 1 week during the preceding 12 months before the interview. A diagram was used to indicate each body area in question. The participants who reported upper-extremity pain or discomfort were asked additional questions about symptom characteristics such as duration and frequency, difficulties in daily activities, and seeking medical care within the past year. Clinical cases based on symptoms and physical examination results were defined as in the study by Sluiter et al (16).

The follow-up questionnaire contained questions on pain or discomfort during the preceding 6 years. The participants were asked to recall if they had pain one 12-month period at a time starting from the preceding 12 months before the follow-up interview until the time period corresponding to the baseline. The 12-month time period corresponding to the baseline was 1991-1992 for those who had had a baseline interview in 1992 and 1992-1993 for those whose baseline was in 1993.

In the present study, information on recalled and reported symptoms was obtained from 464 respondents. Pain or discomfort reported in the baseline questionnaire was compared with the recalled pain or discomfort obtained with the follow-up questionnaire. Forgetting (pain reported at baseline but not recalled in the follow-up) and over-recalling (no pain reported at baseline but pain recalled in the follow-up) of upper-extremity, low-back, and knee pain were examined in relation to personal and occupational characteristics both at baseline and at follow-up. All of the data analyses, including descriptive statistics and Pearson's chi-square tests, were performed with SAS version 8.2 (SAS Institute Inc, Cary, NC, USA).

\section{Results}

More than two-thirds of the participants who had experienced pain or discomfort in the upper extremities, low back, or knee did not recall it 6 years later (tables 1 and 2). Being a clinical case at baseline slightly improved the recall, but forgetting was still very common. A total of $69 \%$ of the clinical cases could not later recall having pain at baseline even if they were then considered as having a specific upper-extremity disorder. Forgetting was slightly less frequent if the participants had reported a history of upper-extremity disorder or a systemic disease at baseline or if they had more than one upper-extremity area affected. Current or recent symptoms strongly influenced pain recall; forgetting approached $100 \%$ among those who had no current or recent symptoms and decreased considerably when symptoms were present at the time of recall. Other characteristics at follow-up that were associated with better accuracy of recall were clinical case status and having symptoms of depression or anxiety.

Over-recalling prior pain was much less common than forgetting (ie, only $9 \%$ of all the participants 
Table 1. Forgetting and over-recalling prior upper-extremity symptoms.

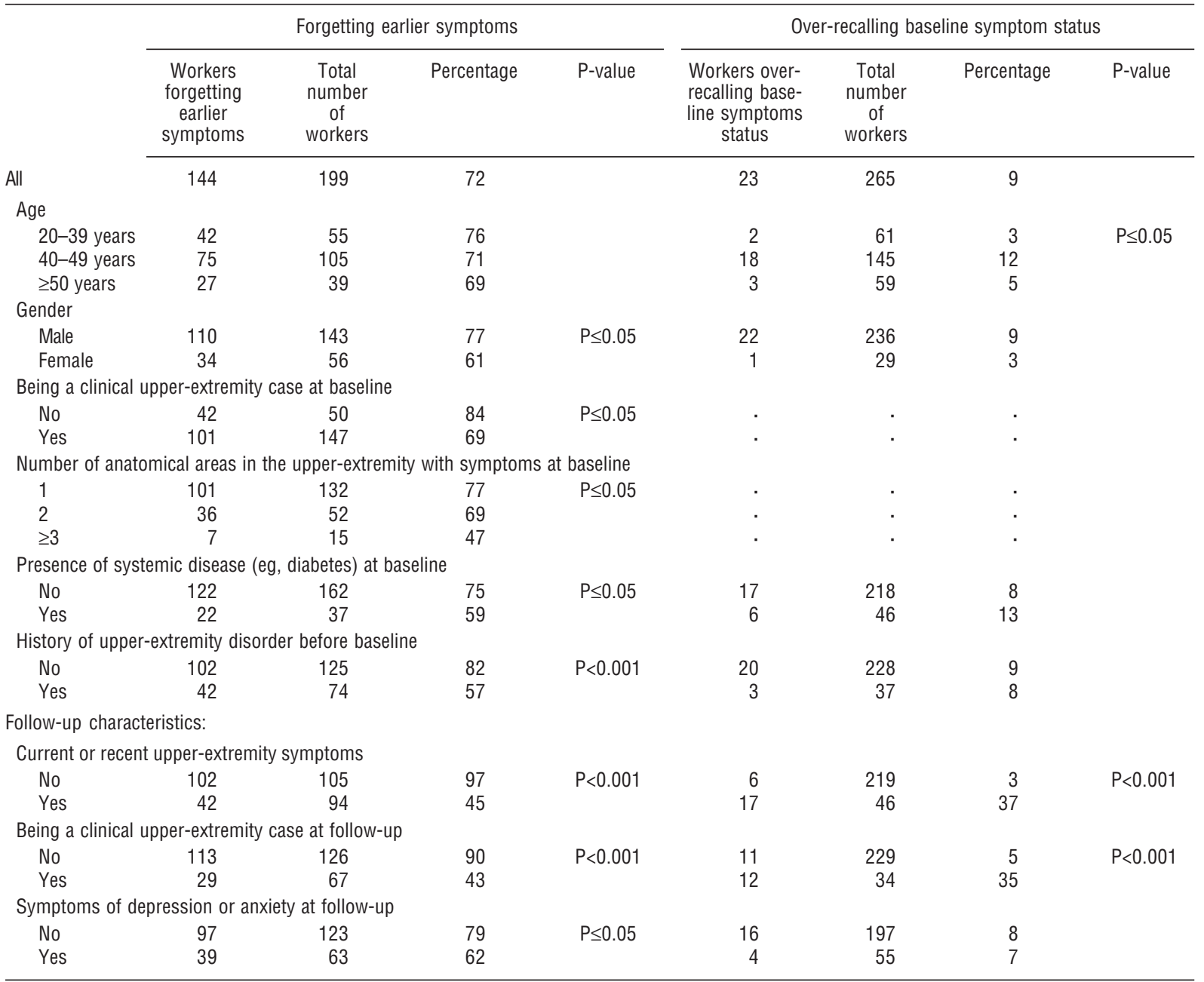

Table 2. Forgetting and over-recalling prior low-back and knee symptoms.

\begin{tabular}{|c|c|c|c|c|c|c|c|c|}
\hline & \multicolumn{4}{|c|}{ Forgetting earlier symptoms } & \multicolumn{4}{|c|}{ Over-recalling baseline symptom status } \\
\hline & $\begin{array}{l}\text { Workers } \\
\text { forgetting } \\
\text { earlier } \\
\text { symptoms }\end{array}$ & $\begin{array}{c}\text { Total } \\
\text { number } \\
\text { of } \\
\text { workers }\end{array}$ & Percentage & P-value & $\begin{array}{c}\text { Workers over- } \\
\text { recalling base- } \\
\text { line symptoms } \\
\text { status }\end{array}$ & $\begin{array}{l}\text { Total } \\
\text { number } \\
\text { of } \\
\text { workers }\end{array}$ & Percentage & P-value \\
\hline \multicolumn{9}{|c|}{ Low back symptoms } \\
\hline All & 74 & 107 & 69 & & 36 & 364 & 10 & \\
\hline \multicolumn{9}{|c|}{ Current or recent symptoms in the low back } \\
\hline No & 53 & 53 & 100 & $P<0.001$ & 5 & 293 & 2 & $P<0.001$ \\
\hline Yes & 21 & 54 & 39 & & 31 & 71 & 44 & \\
\hline \multicolumn{9}{|c|}{ Knee symptoms } \\
\hline All & 32 & 46 & 70 & & 18 & 421 & 4 & \\
\hline \multicolumn{9}{|c|}{ Current or recent symptoms in the knees } \\
\hline No & 26 & 28 & 93 & $P<0.001$ & 1 & 365 & 0.3 & $\mathrm{P}<0.001$ \\
\hline Yes & 6 & 18 & 33 & & 17 & 56 & 30 & \\
\hline
\end{tabular}

recalled having pain they had not reported at baseline. However, over-recalling was also strongly affected by pain at the time of recall; $37 \%$ of those with current symptoms over-recalled their prior pain status, whereas over-recalling occurred for only $3 \%$ of those currently free of symptoms. Over-recalling was particularly common among those with current symptoms but whose symptoms were not continuous (50\%) or did not cause functional impairment (56\%). Neither forgetting nor over-recalling were significantly related to the follow- 
ing factors: functional impairment or seeking medical care due to upper-extremity symptoms at baseline, history of upper-extremity injury before the baseline examination, ethnicity, body mass index, physical activity, alcohol intake, tobacco use, job strain, or work-related physical loading on the upper extremities either at baseline or at follow-up. We performed additional analyses with outcomes with symptoms in all areas (upper extremities, low back and knee) combined, and for lowback and knee pain separately, but the results did not markedly change.

Figure 1 shows in a diagram of how the respondents with or without current or recent upper-extremity symptoms recalled their prior upper-extremity symptoms year by year. Among those who did not have symptoms at the time of recall, the prevalence for recalled symptoms did not exceed 5\% in any of the preceding 12-month periods. The reported prevalence in the baseline questionnaire among these participants was, however, $32 \%$. Regarding those with symptoms at follow-up, all of the 52 participants who correctly recalled having symptoms at baseline had recalled having symptoms every year in between the two time points.

\section{Discussion}

Our study of a cohort of manufacturing workers showed that musculoskeletal pain or discomfort experienced 6 years earlier is rarely recalled accurately. Under-recall- ing, which we would rather call "forgetting" in this study (but which perhaps could also be considered "denying" or "under-reporting"), was very common; most of the participants who had reported pain at baseline were unable to recall it at follow-up. Even the existence of significant pain (based on frequency and duration) or having functional impairment due to symptoms at baseline did not significantly improve the accuracy of recall. It seems that current pain status, particularly the absence of symptoms at the time of recall (or during 12 months preceding it) dictated the recall process; of those who did not have current symptoms, only a few recalled experiencing upper-extremity pain during the preceding 6 years (prevalence being consistently lower than 5\%), although their baseline information indicated a much higher prevalence of symptoms (32\%). Forgetting was considerable even among those with current symptoms (45\%). However, another significant phenomenon was prominent if symptoms were present at the time of recall: over-recall of baseline symptoms. Interestingly, the pattern of over-recalling was not as consistent as that of forgetting; the highest over-recall rates were found among those whose symptoms at the time of recall were not severe in nature (ie, not continuous) and did not cause problems in daily functioning.

Although we have not found any published studies similar to ours focusing on musculoskeletal symptom recall outside clinical settings, earlier studies have shown that the current pain intensity level distorts the recall of prior pain intensity $(1,7,12,13)$.

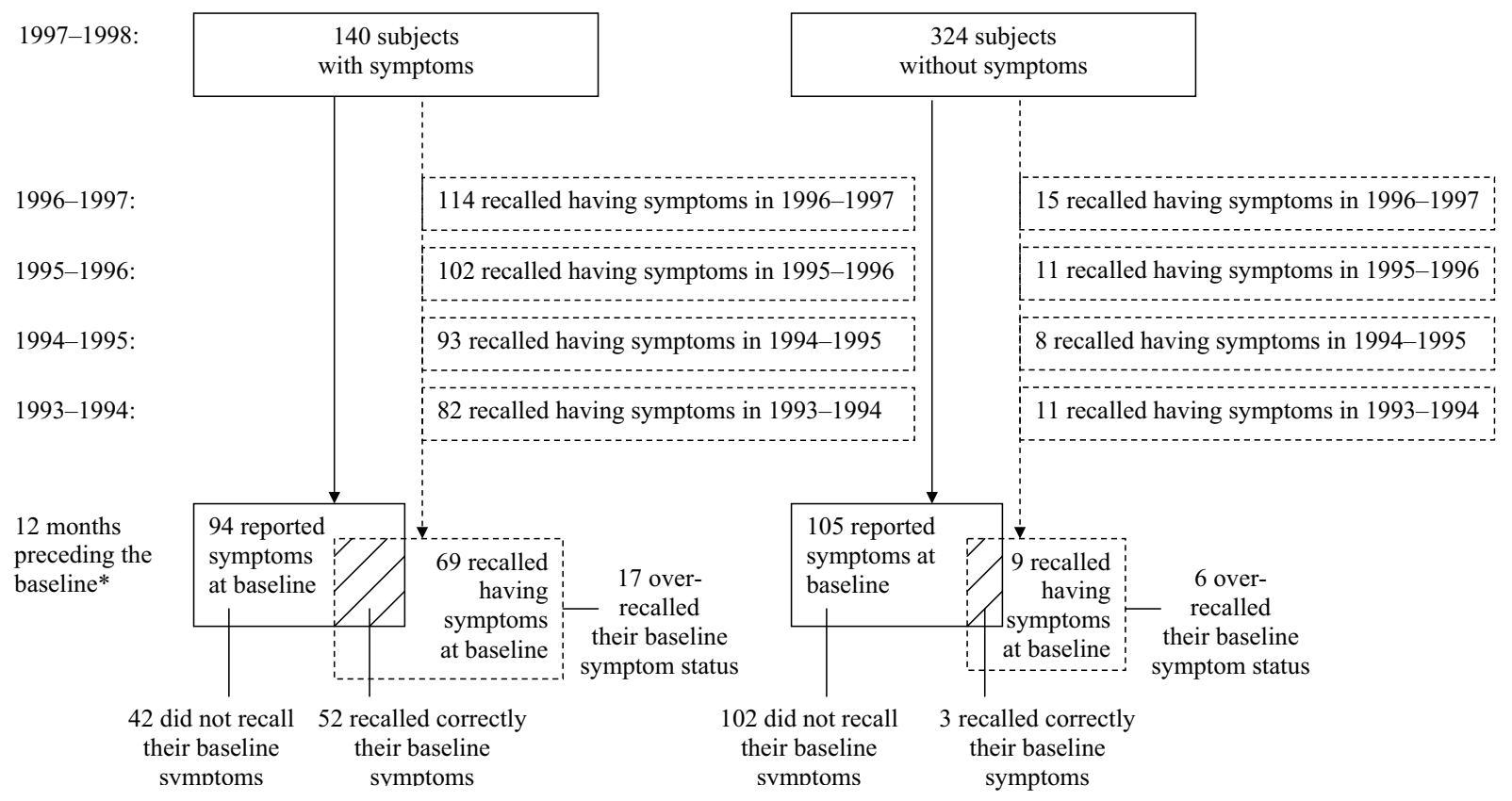

*referring to the time period 1991-1992 for the respondents whose baseline survey was in 1992 and 1992-1993 for those whose baseline survey was in 1993.

Figure 1. Reported and recalled upper-extremity symptoms by symptom status at the time of recall [ie, at follow-up in 1998 (reported symptoms marked with solid line, recalled symptoms with dashed line)] 
Chronicity or the frequent occurrence of pain has been related to poor recall as well (17). It seems that, with time, memories lose their details and events with similar characteristics such as recurrent pain become melded together or are lost (18). Pain recall can be influenced by factors other than pain itself. For instance, emotional status either at the time of the pain experience or pain recall modulates the recall process $(3,19)$. It has been proposed that when the period of recall is short (eg, <1 month), it is the actual experienced pain that best predicts the recall. Over time, the recall process is increasingly influenced by negative emotions in that people seem to be able to recall their pain more accurately if emotions such as anxiety or fear have also been present at the time of the pain experience (19). Persistent pain is often associated with negative emotions such as anxiety and depression (20). In our study, all of the participants who were able to recall their baseline pain correctly also recalled having pain every year between the baseline and the follow-up (ie, their symptoms seemed to be chronic in nature). They also had symptoms of depression and anxiety more often according to the follow-up questionnaire. They were apparently able to recall their pain correctly because of the persistence of their symptoms or because of negative emotions related to their prolonged condition or both.

As it is evident that earlier episodes of pain are easily forgotten, it would be important to be able to assess the length of the time period after which forgetting begins or worsens. Unfortunately our study was unable to answer this question. The history of lifetime pain (ever experienced) is presumably more easily recalled than pain that has occurred only during a limited period of time. Walsh et al (21) showed that, at an interval of 12 months, the question of the history of low-back pain had high repeatability $(\mathrm{k}=0.82$; of those who had a history of low-back pain at baseline, $8 \%$ reported having no such history in the follow-up questionnaire). A history of associated sciatica or disability was less reproducible (21). In the study of Riihimäki et al (22), the follow-up period was 3 years, and $31 \%$ of those who had a history of sciatic pain at baseline reported never having experienced sciatic pain in the follow-up. In a study of Bovenzi \& Zadini (23), the lifetime prevalence of low-back pain differed only very little from the prevalence in the previous 12 months.

The question of the history of lifetime pain is relatively rarely used in modern musculoskeletal disorder research, whereas the 12-month time period seems to be the standard assessment. However, another relatively common pain (ie, chest pain, generally assessed with the WHO Rose chest pain questionnaire)p begins with a question regarding lifetime pain: "Have you ever had any pain or discomfort in your chest?" (24). The WHO Rose chest pain questionnaire is an extensively used screening tool for cardiovascular symptoms particularly in population-based studies. We were unable to find any papers that had evaluated the possible recall error related to the reporting of the history of chest pain. We can only conclude that, if the magnitude of forgetting symptoms such as chest pain is anywhere near to that of upper-extremity, low-back, or knee pain, it will inevitably lead to misclassification of "healthy" people and, hence, to serious underestimation of the prevalence estimates and, at worst, to biased risk estimates. Our own unpublished data from a population-based followup survey of 989 adults in Finland shows that, at baseline, 387 people reported having ever experienced heart or chest pain. However, after 20 years, 164 (42\%) of these 387 persons reported never having pain or discomfort in the chest. Even among those who had reported having rather or very much chest pain at baseline, $21 \%$ forgot in the follow-up that they had ever had such symptoms.

Another implication of our results is related to the question of the onset of the disorder. Identifying the onset of symptoms or a disorder retrospectively is needed when, for instance, the window of therapeutic opportunity is assessed for disorders such as rheumatoid arthritis and, occasionally, even for medicolegal purposes. In their 5-year follow-up study, Amjadi-Begvand et al (25) showed that, among early rheumatoid arthritis patients, the accuracy of recalling the onset of their rheumatoid arthritis symptoms decreased consistently over a period of 5 years. After 70 months, most of the patients $(75 \%)$ were unable to recall the symptom-onset date (to within 1 month) that they had reported at baseline, and over time the underestimation of the duration of the rheumatoid arthritis increased. Patients with longer disease duration, less disease activity, and higher pain levels tended to be less accurate (25). The literature acknowledges a phenomenon called the telescoping effect, which is the temporal displacement of an event so that the event is dated too remotely (backward telescoping) or too recently (forward telescoping) $(5,26$, 27 ). The over-recalling detected in our study could indicate the presence of such backward telescoping; those with current symptoms may have erroneously reported an earlier onset than the actual onset time period.

Our study has some limitations. Because of the small number of women, we were unable to examine factors related to recall separately by gender. However, there was an indication of slightly better recall accuracy among the women than among the men. This study population could also be considered to represent a survivor population with respect to soft-tissue disorders since the workers had been employed, on the average, by the company more than 20 years and had worked in their current jobs more than 10 years (14). We cannot say whether those who were selected out of the workforce 
due to musculoskeletal problems differed from this population in their accuracy of recalling prior symptoms. With respect to loss to follow-up, those who were eligible but did not participate in the follow-up study did not differ from those who participated regarding being an upper-extremity case at baseline. We do not think that the possible difference in the symptom status at followup between the participants and nonparticipants would have biased our main results of the influence of current symptoms on recall.

In conclusion, persons who are no longer symptomatic are not likely to remember their prior pain, even when it has been severe, and those with current symptoms will often over-recall their prior symptom status. We assume, and the literature supports our assumption, that these results can be generalized to symptoms reported for disorders other than only musculoskeletal ones. Recall of past symptoms should not be relied upon when an epidemiologic case definition is constructed or when attempts are made to describe the natural history of a disorder.

\section{Acknowledgments}

This study was funded by the United Auto WorkersChrysler (now Daimler-Chrysler) National Joint Committee on Health and Safety and the United States National Institute for Occupational Safety and Health (NIOSH), grant number R01-OH003514. The contents of this report are solely the responsibility of the authors and do not necessarily represent the official views of NIOSH.

\section{References}

1. Linton SJ, Melin L. The accuracy of remembering chronic pain. Pain. 1982;13:281-5.

2. Eich E, Reeves JL, Jaeger B, Graff-Radford SB. Memory for pain: relation between past and present pain intensity. Pain. 1985;23:375-80.

3. Jamison RN, Sbrocco T, Parris WC. The influence of physical and psychosocial factors on accuracy of memory for pain in chronic pain patients. Pain. 1989;37:289-94.

4. Linton SJ. Memory for chronic pain intensity: correlates of accuracy. Percept Mot Skills. 1991;72:1091-5.

5. Carey TS, Garrett J, Jackman A, Sanders L, Kalsbeek W. Reporting of acute low back pain in a telephone interview. Identification of potential biases. Spine. 1995;20:787-90.

6. Bolton JE. Accuracy of recall of usual pain intensity in back pain patients. Pain. 1999;83:533-9.

7. de Wit R, van Dam F, Hanneman M, Zandbelt L, van Buuren A, van der Heijden K, et al. Evaluation of the use of a pain diary in chronic cancer pain patients at home. Pain. 1999;79:89-99.
8. Salovey P, Smith AF, Turk DC, Jobe JB, Wills GB. The accuracy of memory for pain: not so bad most of the time. Am Pain Soc J. 1993;2:184-91.

9. Barsky AJ. Forgetting, fabricating, and telescoping: the instability of the medical history. Arch Intern Med. 2002;162:9814.

10. Schmier JK, Halpern MT. Patient recall and recall bias of health state and health status: expert review of pharmacoeconomics \& outcomes research. 2004;4:89-93.

11. Dawson EG, Kanim LE, Sra P, Dorey FJ, Goldstein TB, Delamarter RB, et al. Low back pain recollection versus concurrent accounts: outcomes analysis. Spine. 2002;27:984-93.

12. Smith WB, Safer MA. Effects of present pain level on recall of chronic pain and medication use. Pain. 1993;55:355-61.

13. Brauer C, Thomsen JF, Loft IP, Mikkelsen S. Can we rely on retrospective pain assessments? Am J Epidemiol. 2003; 157:552-7.

14. Punnett L. Ergonomic stressors and upper extremity disorders in vehicle manufacturing: cross sectional exposure-response trends. Occup Environ Med. 1998;55:414-20.

15. Gold JE, Park JS, Punnett L. Work routinization and implications for ergonomic exposure assessment. Ergonomics. 2006;49:12-27.

16. Sluiter JK, Rest KM, Frings-Dresen MH. Criteria document for evaluating the work-relatedness of upper-extremity musculoskeletal disorders. Scand J Work Environ Health. 2001;27 suppl 1:1-102.

17. Erskine A, Morley S, Pearce S. Memory for pain: a review. Pain. 1990;41:255-65.

18. Means B, Nigam A, Zarrow M, Loftus EF, Donaldson MS. Autobiographical memory for health-related events. Hyattsville (MD): National Center for Health Statistics; 1989. Vital health stat 6 .

19. Gedney JJ, Logan H. Memory for stress-associated acute pain. J Pain. 2004;5:83-91.

20. Gureje O, Von Korff M, Simon GE, Gater R. Persistent pain and well-being: a World Health Organization study in primary care. JAMA. 1998;280:147-51.

21. Walsh K, Coggon D. Reproducibility of histories of low-back pain obtained by self-administered questionnaire. Spine. 1991;16:1075-7.

22. Riihimäki H, Viikari-Juntura E, Moneta G, Kuha J, Videman $\mathrm{T}$, Tola S. Incidence of sciatic pain among men in machine operating, dynamic physical work, and sedentary work: a three-year follow-up. Spine. 1994;19:138-42.

23. Bovenzi M, Zadini A. Self-reported low back symptoms in urban bus drivers exposed to whole-body vibration. Spine. 1992;17:1048-59.

24. Rose GA. The diagnosis of ischaemic heart pain and intermittent claudication in field surveys. Bull World Health Organ. 1962;27:645-58.

25. Amjadi-Begvand S, Khanna D, Park GS, Bulpitt KJ, Wong WK, Paulus HE. Dating the "window of therapeutic opportunity" in early rheumatoid arthritis: accuracy of patient recall of arthritis symptom onset. J Rheumatol. 2004;31:1686-92.

26. Thompson CP, Skowronski JJ, Lee DJ. Telescoping in dating naturally occurring events. Mem Cognit. 1988;16:461-8.

27. Bradburn NM. Temporal representation and event dating. In: Stone AA, Turkkan JS, Bachrach CA, Jobe JB, Kurtzman HS, Cain VS, editors. The science of self-report: implications for research and practice. Mahwah (NJ): Lawrence Erlbaum Associates Inc; 2000. p 49-61.

Received for publication: 8 December 2005 